\title{
A proteomic approach to the development of DIVA ELISA distinguishing pigs infected with Salmonella Typhimurium and pigs vaccinated with a Salmonella Typhimurium- based inactivated vaccine
}

\author{
Jan Gebauer ${ }^{1,2^{*}}$, Hana Kudlackova ${ }^{1}$, Marcel Kosina ${ }^{3}$, Kamil Kovarcik' ${ }^{4}$, Radek Tesarik', Alena Osvaldova ${ }^{1,5}$,
} Martin Faldyna ${ }^{1}$ and Jan Matiasovic ${ }^{1}$

\begin{abstract}
Background: Salmonella enterica serovar Typhimurium is one of the most common enteropathogenic bacteria found in pigs in Europe. In our previous work, we demonstrated the protective effects in suckling piglets when their dams had been vaccinated with an S. Typhimurium-based inactivated vaccine. This study is focused on a procedure leading to serological discrimination between vaccinated and infected pigs. As we supposed, distinct environment during natural infection and in bacterial cultures used for vaccine preparation led to a slightly different spectrum of expressed S. Typhimurium proteins. The examination of porcine antibodies produced after the experimental infection with $S$. Typhimurium or after vaccination with $S$. Typhimurium-based inactivated vaccine by affinity chromatography and mass spectrometry revealed differences in antibody response applicable for serological differentiation of infected from vaccinated animals.

Results: Antibodies against Salmonella SipB, SipD and SseB proteins were detected at much higher levels in postinfection sera in comparison with control and post-vaccination sera. On the other hand, proteins BamB, OppA and a fragment of FliC interacted with antibodies from post-vaccination sera with a much higher intensity than from control and post-infection sera. In addition, we constructed ELISA assays using post-infection antigen - SipB protein and post-vaccination antigen - FliC-fragment and evaluated them on a panel of individual porcine sera.

Conclusions: The analysis of antibody response of infected and vaccinated pigs by proteomic tools enabled to identify S. Typhimurium antigens useful for distinguishing infected from vaccinated animals. This approach can be utilized in other challenges where DIVA vaccine and a subsequent serological assay are required, especially when genetic modification of a vaccine strain is not desirable.
\end{abstract}

Keywords: Porcine, Salmonella Typhimurium, DIVA vaccine, Recombinant protein, Mass spectrometry

\footnotetext{
*Correspondence: gebauer@vri.cz.

'Department of Immunology, Veterinary Research Institute, Hudcova296/70, 62100 Brno, Czech Republic

2Department of Experimental Biology, Faculty of Science, Masaryk University, Kotlarska267/2, 61137 Brno, Czech Republic

Full list of author information is available at the end of the article
} 


\section{Background}

Infections caused by non-typhoid Salmonella enterica serovar Typhimurium (Salmonella Typhimurium) constitute a persistent problem in human and veterinary medicine. Salmonella Typhimurium is the most frequent serotype found in pigs. Contaminated pork and porcine products are thus a source of infection for human consumers [1]. A possible way to moderate the Salmonella burden in pigs is vaccination. A successful and widely used vaccine should allow distinguishing vaccinated animals from those that were naturally infected, so-called DIVA approach (Differentiating Infected from Vaccinated individuals) [2]. Available diagnostic serological tests for the evaluation of Salmonella infections in pigs are based on measurements of the level of antibodies induced by $\mathrm{O}$-antigens, the outer segment of bacterial lipopolysaccharide (LPS) [3]. These assays do not allow us to distinguish infected and vaccinated animals when Salmonella strain without any deletion in genes responsible for lipopolysaccharide formation is used for vaccination [4]. On the other hand, LPS plays a role as an inducer of the immune response, which might be beneficial for the vaccination itself $[5,6]$. Selke et al. [7] introduced a live negative-marker vaccine based on Salmonella Typhimurium strain with deleted gene for the outer membrane protein $o m p D$. Bearson et al. [8] prepared a similar attenuated live negative-marker vaccine with a deletion of multiple small regulatory RNAs combined with $r f a H$ mutation. However, using a genetically modified live bacterial strain as a vaccine may be questionable because of current regulation and public non-acceptance of genetically modified organisms in Europe.

With an unmodified inactivated Salmonella Typhimurium-based vaccine for pigs developed in our previous work we achieved a similar level of protectivity for suckling piglets [9] as Selke et al. [7]. In this study, we extend the development of this vaccine to include DIVA testing. We took advantage of the fact that Salmonella express virulence factors (proteins from SPIs - Salmonella pathogenicity islands) in an environment-dependent manner. We thus expected differences in bacterial protein expression under in vitro and in vivo conditions. We analysed the antibody response to the vaccine based on inactivated Salmonella Typhimurium cultivated in vitro and the antibody response of animals infected with live bacteria.

Based on this, we introduced a method for discovering Salmonella proteins able to induce condition-specific antibody production, which allows us to serologically distinguish animals that were vaccinated from those infected with Salmonella Typhimurium.

\section{Methods}

\section{Bacterial strain}

Salmonella enterica serovar Typhimurium phage type DT104 strain (strain number 1A5, from bacterial collection at Veterinary Research Institute, originally isolated from healthy sow), hereinafter referred to as Salmonella Typhimurium, was used in this experiment. Bacteria were cultivated overnight at $37{ }^{\circ} \mathrm{C}$ in Miller's LB Broth Base (Invitrogen, USA) or brain heart infusion (BHI) broth (Oxoid, UK) for certain analyses as described below.

\section{Vaccination and infection of pigs}

Three groups of animals consisting of twelve white mixed-breed piglets (bought from a commercial stud) weaned 21 days after birth were used in the experiment. Pigs in the first group remained serologically negative for anti-Salmonella antibodies (determined by Pig Screen ELISA, Qiagen, Germany). Another twelve animals were orally infected one week after housing with $1 \times 10^{8}$ CFU of Salmonella Typhimurium grown in BHI medium and blood was collected 28 days after the infection. The last group of animals was vaccinated intramuscularly into the neck with $1 \mathrm{ml}$ of a vaccine prepared from $1 \times 10^{9} \mathrm{CFU}$ of Salmonella Typhimurium grown in BHI medium, inactivated with formaldehyde and adjuvanted with Montanide ISA50V2 (Seppic, France). The first dose was administered one week after housing and the second dose two weeks later. Blood was collected 14 days after the second dose of a vaccine.

\section{Antibody fraction preparation}

Serum samples from three random animals from each group were pooled together and IgG fractions were isolated using Protein G columns (HiTrap Protein G HP, GE Healthcare, UK) according to the manufacturer's protocol. Affinity chromatography was performed on an FPLC instrument (Pharmacia, Sweden).

\section{Antigen preparation}

The same procedure of preparing bacterial protein lysate was used for the comparison of Salmonella Typhimurium protein expression when cultivated in LB or BHI medium using MS and for immunoaffinity chromatography.

Salmonella Typhimurium was grown overnight at $37^{\circ} \mathrm{C}$ in LB and BHI medium, as noted above. The culture was then centrifuged $(3,500 \times \mathrm{g}, 10 \mathrm{~min})$, and a cell pellet was washed 3 times in PBS (Dulbecco's, Lonza, Switzerland). The cell pellet was resuspended in PBS and sonicated (Sonopuls HD 3100, Bandelin, Germany) with zirconia/silica beads (BioSpec Products, USA). The sonicate was centrifuged at $20,000 \times \mathrm{g}$ and the supernatant with proteins was taken. The pellet was then resuspended in $8 \mathrm{M}$ urea (Serva, Germany), 0.1 \% SDS (Carl Roth, Germany), $2 \%$ Triton X-100 (Serva, Germany) and $25 \mathrm{mM}$ triethylammonium bicarbonate (Sigma-Aldrich, USA) and centrifuged at $20,000 \times g$ again. The supernatant was mixed 
with the supernatant from the previous step and together used as an antigen for subsequent analysis.

\section{Immunoaffinity chromatography}

IgGs from all three sample groups were covalently bound to CNBr-activated Sepharose (GE Healthcare, UK) according to the manufacturer's protocol. Sepharose with bound immunoglobulins was loaded into 3 plastic cartridges (BioRad, USA) creating liquid chromatography columns. The same antigen (protein lysate from Salmonella Typhimurium grown in LB) was used for all three IgG columns - column with antibodies from negative control animals, from vaccinated animals and from infected animals. $20 \mathrm{mM}$ sodium phosphate buffer, pH 7.0 (Serva, Germany) served as a binding buffer. $0.1 \mathrm{M}$ glycine- $\mathrm{HCl}$ (Serva, Germany), $\mathrm{pH} 2.7$ was used for elution of bound proteins and $1 \mathrm{M}$ Tris- $\mathrm{HCl}$ (Carl Roth, Germany), pH 9.0, was added to collection tubes for $\mathrm{pH}$ adjustment. Protein eluates were then used for analysis by mass spectrometry.

\section{LC-MS/MS analysis}

$100 \mu \mathrm{g}$ of proteins (measured with Pierce ${ }^{\mathrm{Tm}}$ BCA Protein Assay Kit, Thermo Scientific, USA) was used for each sample preparation with the FASP (filter-aided sample preparation) method [10]. Each sample was washed five times with $8 \mathrm{M}$ urea (Serva, Germany) in Vivacon 500 centrifugal tubes (Sartorius Stedim, Germany) with 10,000 MWCO membrane filter. Dithiothreitol (10 mM, Sigma-Aldrich, USA) and iodoacetamide (50 mM, Serva, Germany) in triethylammonium bicarbonate buffer (25 mM, Sigma-Aldrich, USA) were used for reduction and alkylation, respectively. Proteins were then digested with trypsin (Promega, USA) in 1:50 ratio, for one hour, at $37{ }^{\circ} \mathrm{C}$ and then overnight at $25{ }^{\circ} \mathrm{C}$. After centrifugation, the eluate with digested peptides was evaporated (DNA120 SpeedVac, Thermo Savant, USA), peptide pellet was resuspended in $0.1 \%$ aqueous formic acid (Sigma-Aldrich, USA) which serves as a mobile phase for liquid chromatography (UltiMate 3000 RSLCnano, Dionex -Thermo Scientific, USA). For separation and elution of peptides, 2-h gradient with increasing (0 min 4. \%, $4 \mathrm{~min}-4 \%$, $98 \mathrm{~min}-45 \%$, $98.5 \mathrm{~min}-90 \%$, $112 \mathrm{~min}-90 \%, 112.5 \mathrm{~min}-4 \%, 120 \mathrm{~min}-4 \%)$ concentrations of acetonitrile $(0.1 \%$ formic acid in acetonitrile, Sigma-Aldrich, USA) at a flow rate of $300 \mathrm{nl} / \mathrm{min}$ was used. Peptides were separated on a $25 \mathrm{~cm}$ column (Acclaim PepMap RSLC C18, $2 \mu \mathrm{m}, 100 \AA$, $75 \mu \mathrm{m}$ I.D., Thermo Scientific, USA). uHPLC was connected to EASY-Spray ion source and Orbitrap Velos Pro mass spectrometer (Thermo Scientific, USA). A survey scan over the $\mathrm{m} / \mathrm{z}$ range $390-1700$ was used to identify protonated peptides with charge states of at least 2, which were automatically selected for data-dependent MS/MS analysis and fragmented by collision with helium gas. Ten fragment mass spectra after each full scan were recorded. Measured spectra were then searched using Proteome Discoverer (version 1.4, Thermo Scientific, USA) software with Mascot (Matrix Science, USA) or Sequest HT (Thermo Scientific, USA) as a searching algorithm. Oxidation of methionine as a dynamic, and carbamidomethylation of cysteine as a static modification was used. Precursor and fragment mass tolerances were set up as $10 \mathrm{ppm}$ and $0.5 \mathrm{Da}$, respectively. Swiss-Prot database for Salmonella strain was used (based on 2014/ 06 release) in Mascot and Uniprot database for Salmonella strain (from 2013/04) was used in Sequest HT. Only peptides with a false discovery rate less than 0.05 were considered as well identified. Proteins that were uniquely found in each sample - proteins bound to the IgG fraction from sera from either vaccinated or infected animals, but not from control pigs were then pinpointed as candidate antigens for serological discrimination.

\section{Recombinant protein preparation and its purification}

Appropriate primers (Tab. 1) for each selected protein were designed using NCBI/Primer-BLAST. PCR reaction with Salmonella DNA (supernatant from washed cell culture heated in distilled water for $10 \mathrm{~min}$ at $95^{\circ} \mathrm{C}$ ) as a template and Taq polymerase (Plain PP Master Mix, Top Bio, Czech Republic) was carried out. PCR products were purified (MinElute PCR Purification Kit, Qiagen, Germany), then cloned into the pTrcHis-TOPO vector (Invitrogen, USA) and recombinant proteins were expressed in TOP10 One Shot chemically competent $E$. coli cells (Invitrogen, USA). IPTG was used as an inducer of the expression. Recombinant proteins with $\mathrm{N}$-terminal His-Tag were isolated on HiTrap Chelating HP columns (GE Healthcare, UK) with attached nickel ions. Briefly, an overnight $E$. coli culture in LB broth (Invitrogen, USA) was centrifuged and washed in PBS (Dulbecco's, Lonza, Switzerland) and cells were disrupted by sonication (Sonopuls HD 3100, Bandelin, Germany). After centrifugation, supernatant with proteins was transferred to a binding buffer $(0.02 \mathrm{M}$ sodium phosphate, $0.5 \mathrm{M} \mathrm{NaCl}, 5 \mathrm{mM}$ imidazole, $\mathrm{pH}$ 7.4) using desalting columns (PD-10, Sephadex G-25 M, GE Healthcare, UK). Metal-chelate affinity chromatography was performed on an FPLC system (Pharmacia, Sweden). After washing with a buffer containing an increased concentration of imidazole $(40 \mathrm{mM})$, His-Tagged proteins were finally eluted with the elution buffer $(0.02 \mathrm{M}$ sodium phosphate, $0.5 \mathrm{M}$ $\mathrm{NaCl}$ and $250 \mathrm{mM}$ imidazole, $\mathrm{pH}$ 7.4).

If further purification was needed, we performed a preparative electrophoresis in a polyacrylamide gel (Model 491 Prep Cell, BioRad, USA) according to the manufacturer's protocol. For protein expression and purity verification, SDS-PAGE and western blot with a mouse anti- 
Table 1 Primer pairs of DNA sequences for used proteins (or polypeptide fragments) and their expected molecular weight including HisTag

\begin{tabular}{|c|c|c|c|}
\hline Protein & Forward primer 5'-3' & Reverse primer 5'-3' & Expected protein molecular weight \\
\hline BamB & ATGCAATTGCGTAAATTACTTCTGCC & TTAACGCGTAATCGCGTAGACC & $45.8 \mathrm{kDa}$ \\
\hline FliC-frag & ACGCTGAATGTGCAACAAAAATATAAGGTC & ACCTTCGGCTTTACTTGCAGCG & $25.8 \mathrm{kDa}$ \\
\hline MetQ & ATGGCGTTCAAATTCAAAACCTITGCGG & TTACCAGCCTITCACCGCGCC & $33.3 \mathrm{kDa}$ \\
\hline Prgl & ATGGCAACACCTTGGTCAGGCTATC & TTAACGGAAGTTCTGAATAATGGCAGCATC & $12.7 \mathrm{kDa}$ \\
\hline $\mathrm{SipC}$ & ATGTTAATTAGTAATGTGGGAATAAATCCCGCC & TTAAGCGCGAATATTGCCTGCGAT & $46.8 \mathrm{kDa}$ \\
\hline SipD & ATGCTTAATATTCAAAATTATTCCGCT & TTATCCTTGCAGGAAGCTITT & $41.0 \mathrm{kDa}$ \\
\hline SseB & ATGTCTTCAGGAAACATCTTATGGGGAA & TCATGAGTACGTITCTGCGCTATC & $25.4 \mathrm{kDa}$ \\
\hline SurA & ATGAAGAACTGGAAAACGCTGCTTC & TTAGTTACTCAAAATCTTAACGTAAGCGCTG & $51.1 \mathrm{kDa}$ \\
\hline $\operatorname{Sip} B$ & ATGGTAAATGACGCAAGTAGCATTAGCCG & TTATGCGCGACTCTGGCGCAGAATAAAAC & $66.3 \mathrm{kDa}$ \\
\hline OppA & ATGTCTAACATCACGAAAAAAAGTTTG & TTAATGTTTGATAATATATAAGTITTTCACATAAAT & $65.1 \mathrm{kDa}$ \\
\hline
\end{tabular}

HisTag antibody (Pierce, Thermo Scientific, USA) was used.

\section{Western blot}

Purified recombinant proteins were resolved on a $10 \%$ SDS-PAGE gel and transferred to PVDF membranes (Amersham, GE Healthcare, UK), which were subsequently blocked in $1 \%$ casein hydrolysate (Imuna, Czech Republic) overnight at $4{ }^{\circ} \mathrm{C}$. The membranes were incubated with $100 \times$ diluted porcine sera for one hour at room temperature (RT), washed in PBS with $0.05 \%$ Tween-20 (PBS/T, Serva, Germany) and then incubated (one hour, RT) with goat anti-pig IgG conjugated with HRP (Bethyl Laboratories, USA). After a washing step, the protein bands were visualized with diaminobenzidine (Sigma-Aldrich, USA). Western blots were performed with group-specific pooled sera samples.

\section{ELISA assay}

Purified recombinant proteins in $0.05 \mathrm{M}$ sodium carbonate-bicarbonate buffer $\left(\mathrm{pH}\right.$ 9.6) were coated $\left(4{ }^{\circ} \mathrm{C}\right.$ overnight) on microtiter plates (MaxiSorp, Nunc, Denmark) that were blocked afterwards with $1 \%$ casein hydrolysate in PBS/T (30 min). The plates were subsequently incubated (one hour, RT) with $100 \times$ diluted porcine sera, washed with $\mathrm{PBS} / \mathrm{T}$ and then incubated (one hour, RT) with goat anti-pig IgG conjugated with HRP (Bethyl Laboratories, USA). After a washing step, the colour was developed by adding the chromogenic substrate TMB Complete (Test-line, Czech Republic) and the reaction was stopped with $2 \mathrm{M}$ sulfuric acid (Penta, Czech Republic). The absorbance was measured at $450 \mathrm{~nm}$ on Synergy H1 (Biotek, USA). IgG antibody response to LPS was determined by commercially available Pig Screen ELISA (Qiagen, Germany). The individual serum samples were tested in duplicate by ELISA. Positive thresholds in time course graphs were counted as an average plus three standard deviations of all control samples and time zero samples of respective groups [11]. Positive thresholds for LPS commercial kit were provided by a manufacturer.

\section{Statistical evaluation}

Densities of western blot bands were calculated using ImageJ software (ver. 1.48). Differences between groups in ELISA assays were evaluated by ANOVA with Dunn's post test using GraphPad Prism (ver. 5). All graphs were also made using GraphPad Prism software (GraphPad Software, USA).

\section{Results}

\section{Protein expression profiles in different media}

At first, we examined protein expression profiles of Salmonella growing in different cultivation media. LB broth was used as a standard growth medium for cultivation of bacteria. For comparison, brain heart infusion (BHI), a highly nutritious medium was used as a vaccine preparation medium when the vaccine was proved to be protective [9]. From the whole-proteome mass spectrometry analysis, we observed the differences in LB and BHI cultures in relative quantities of several proteins that belong to the SPI-1 and SPI-2 loci which are important for the virulence of Salmonella. Relative quantification was attained using spectral counting and, in Tab. 2, it is shown as PSMs (peptide spectrum matches) for all peptides which identify the protein. Since PSMs depend on the length of a protein, the comparison of PSM value for the same protein between each medium is more informative rather than the absolute number. Note that the shown proteins are only representatives of the SPI- 1 and SPI-2 loci and not the complete list.

All selected SPI-1 proteins were expressed in much higher amounts in LB medium than in BHI medium while proteins belonging to the SPI-2 locus were not highly expressed in either of the media. Flagellar proteins that also play an important role in the first stages 
Table 2 Several selected proteins belonging to SPI-1 and/or SPI-2 locus and their relative amounts produced by $\mathrm{S}$. Typhimurium grown in LB and $\mathrm{BH}$ medium, respectively

\begin{tabular}{lllll}
\hline Locus & Protein & $\begin{array}{l}\text { Accession } \\
\text { number }\end{array}$ & & PSMs \\
\cline { 5 - 5 } & & LB & BHI \\
\hline SPI-1 & SipA & P0CL52 & 37 & 2 \\
SPI-1 & SipB & Q56019 & 31 & 4 \\
SPI-1 & SipC & P0CL47 & 50 & 3 \\
SPI-1 & SipD & Q56026 & 10 & 0 \\
SPI-1 & Prgl & P41784 & 5 & 2 \\
SPI-1 & SptP & P74873 & 20 & 0 \\
SPI-1 & SopA & Q8ZNR3 & 16 & 5 \\
SPI-1 & SopB & O30916 & 38 & 0 \\
SPI-1 & SopE2 & Q7CQD4 & 5 & 0 \\
SPI-1 and-2 & SteA & Q8ZPD7 & 0 & 0 \\
SPI-1 and-2 & SopD & P40722 & 5 & 0 \\
SPI-2 & SopD2 & Q8ZQC8 & 3 & 0 \\
SPI-2 & SseB & Q7BVH7 & 0 & 3 \\
SPI-2 & PipB & Q8ZQ59 & 0 & 1 \\
SPI-2 & PipB2 & Q8ZMM8 & 4 & 0 \\
flagellar proteins & FliC & P06179 & 132 & 77 \\
& FljB & P52616 & 107 & 61 \\
reference proteins & PepN & Q8ZQ76 & 48 & 39 \\
& GapA & P0A1P0 & 185 & 279 \\
\hline & & & &
\end{tabular}

of an invasion were expressed in both media in high amounts. Proteins PepN (Aminopeptidase N) and GapA (Glyceraldehyde-3-phosphate dehydrogenase A) are mentioned only as reference proteins.

\section{Mass spectrometry analysis of immunoaffinity chromatography samples}

Salmonella protein lysates preparations and consecutive immunoaffinity chromatography analyses resulted in three sets of samples for MS identification (Salmonella proteins bound to antibodies from control, infected or vaccinated animals). While most of the proteins were identified in all three samples in similar proportion, we found several proteins that were identified in relatively higher amounts in "post-infection" or "post-vaccination" fraction (Tab. 3). Relative amounts are shown as peptide spectrum matches (PSMs). Proteins SipB and SipC from SPI-1 were found in eluates from the column with postinfection sera while several proteins (OppA, BamB and SurA) were predominantly bound to antibodies in postvaccination sera. We also included the identification of proteins that were chosen for their involvement in the infection process (SipD, SseB, PrgI and FliC) rather than from immunoaffinity chromatography/MS analysis only.

\section{Recombinant protein preparation}

All proteins listed in Table 3 were prepared as recombinant proteins in an $E$. coli expression system. The sequences of all proteins containing signal peptides at their $\mathrm{N}$-termini were designed without this peptide. Otherwise, they possessed an identical sequence as naturally occurring in $S$. Typhimurium except FliC protein. Due to an inability of full-length protein expression, flagellar protein FliC was expressed only as a $22 \mathrm{kDa}$ fragment. The expression of proteins was endorsed by SDS-PAGE and confirmed by western blot with an anti-HisTag antibody (Fig. 1 and Additional file 1: Figure S1). Four out of ten selected proteins (MetQ, SurA, PrgI, SipC) were not expressed in sufficient amount or purity and were not included in subsequent serological testing.

\section{Serological testing Western blot}

Purified recombinant proteins were then used as antigens for serological analysis. After western blot, PVDF membranes were incubated with pooled serum samples from three animals of each group (control, infected and vaccinated animals). As shown in Fig. 2, proteins SipB, SipD and SseB presumed to be "post-infection" antigens were detected by sera of infected animals with a higher intensity than by sera of control or vaccinated animals. On the other hand, proteins BamB, FliC-frag and OppA, supposed to be "post-vaccination" antigens, were detected by sera of vaccinated pigs with a higher intensity than by sera of control or infected animals. Anti-HisTag antibody was used as a positive control for recombinant protein presence (in Fig. 2D).

\section{ELISA assay}

Based on western blot results, proteins SipB and FliCfrag were selected for testing specific antibody response of individual animals by ELISA method. Serum samples from 12 animals in each group were used for measuring antibody levels against recombinant SipB and FliC-frag proteins.

Antibody levels against SipB protein in sera of control animals had the values of absorbance between 0.062 and 0.126 ; sera of vaccinated animals had the values of absorbance between 0.075 and 0.147 . The difference in absorbance between these two groups was not statistically significant. Sera of infected pigs had the values of absorbance between 0.248 and 1.056. These values were significantly different $(\mathrm{P}<0.01)$ from the two previous groups of animals.

Antibody levels against FliC-frag proteins in sera of control animals had the values of absorbance between 0.018 and 0.271; sera of infected animals had the values of absorbance between 0.060 and 0.891 . The difference in absorbance between these two groups was not statistically 
Table 3 Identification and relative quantification of chosen proteins in terms of Peptide Spectrum Matches for all three runs

\begin{tabular}{|c|c|c|c|c|}
\hline \multirow[t]{2}{*}{ Protein name } & \multirow{2}{*}{$\begin{array}{l}\text { Accession } \\
\text { number }\end{array}$} & \multicolumn{3}{|c|}{ PSMs (peptide spectrum matches) } \\
\hline & & Control & Post-infection & Post-vaccination \\
\hline Cell invasion protein SipB & Q56019 & $3,0,22$ & $29,20,27$ & $13,7,25$ \\
\hline Cell invasion protein SipC & POCL47 & $5,1,22$ & $30,33,31$ & $7,18,26$ \\
\hline D-methionine-binding lipoprotein MetQ & Q8ZRN1 & $3,1,0$ & $12,5,11$ & $0,0,9$ \\
\hline Periplasmic oligopeptide-binding protein OppA & P06202 & $7,0,0$ & $31,0,0$ & $25,16,14$ \\
\hline Outer membrane protein assembly factor BamB & H9L451 & $0,2,0$ & $0,0,7$ & $7,10,0$ \\
\hline Chaperone SurA & Q7CR87 & $1,5,1$ & $2,0,21$ & $21,15,15$ \\
\hline Cell invasion protein SipD & Q56026 & $7,3,0$ & $4,15,0$ & $4,14,1$ \\
\hline Secreted effector protein SseB & Q7BVH7 & $0,0,0$ & $2,0,0$ & $1,0,1$ \\
\hline Protein Prgl & P41784 & $0,2,0$ & $1,2,0$ & $2,0,1$ \\
\hline Flagellin FliC & P06179 & $3,22,17$ & $23,29,26$ & $19,17,63$ \\
\hline
\end{tabular}

significant. Sera of vaccinated pigs had the values of absorbance between 1.727 and 2.159. These values were significantly different $(\mathrm{P}<0.01)$ from two previous groups of animals (Fig. 3). Hence, proteins SipB and FliC-frag may serve as marker proteins applicable for serological discrimination between vaccinated pigs, pigs after natural infection and pigs without any contact with $S$. Typhimurium.

When compared to a non-infected group, from graphs showing the time course of antibody response (Fig. 4) we

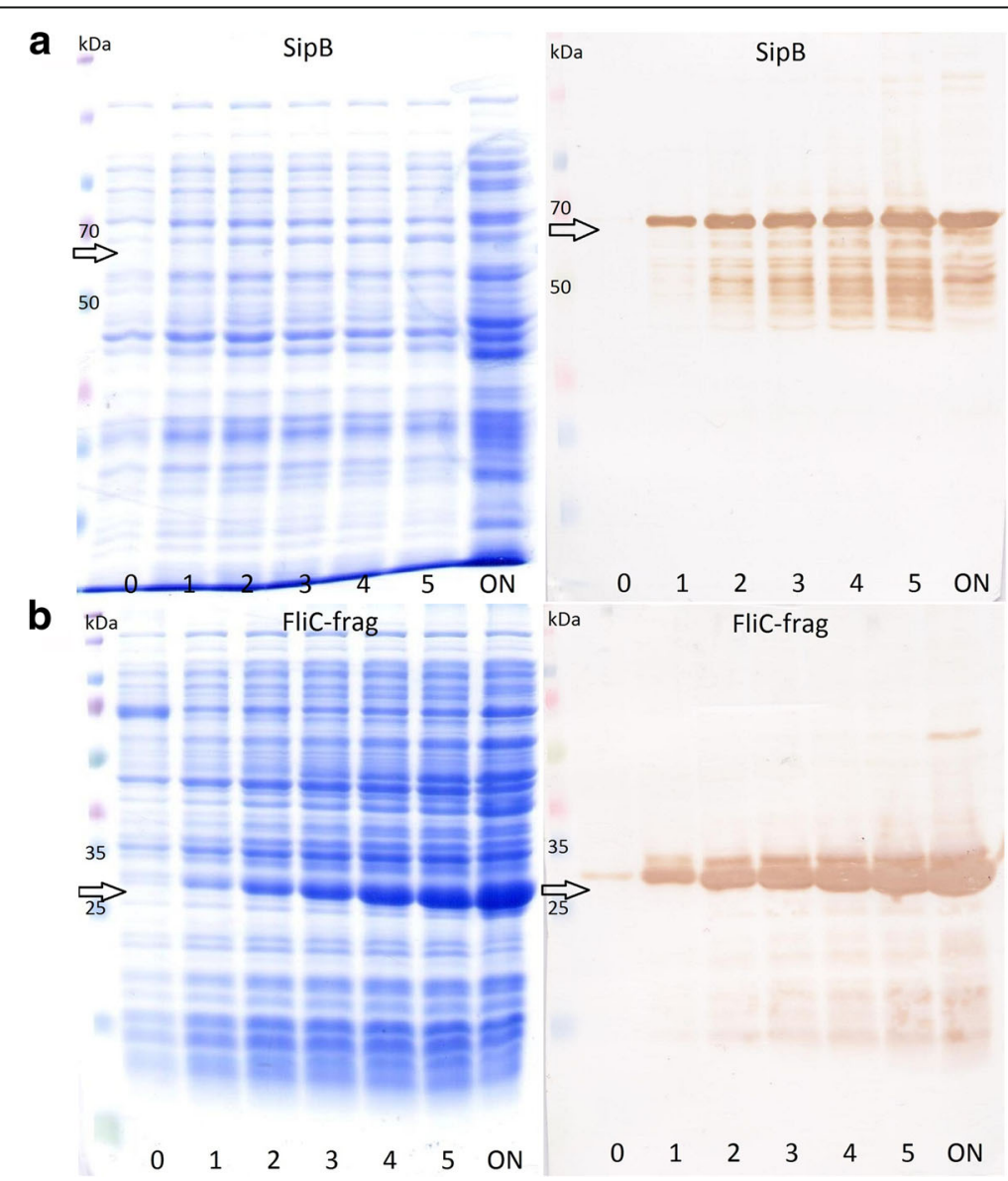

Fig. 1 Recombinant proteins expressions. Coomassie-stained gels and western blots of SipB (a) and FliC-frag (b) proteins. Time scale expression is shown in hours post induction ( $0-5 \mathrm{~h}, \mathrm{ON}$ - overnight). Arrows indicate the expected molecular weight of a product. Expression of other proteins can be found in Additional file 1: Figure S1 


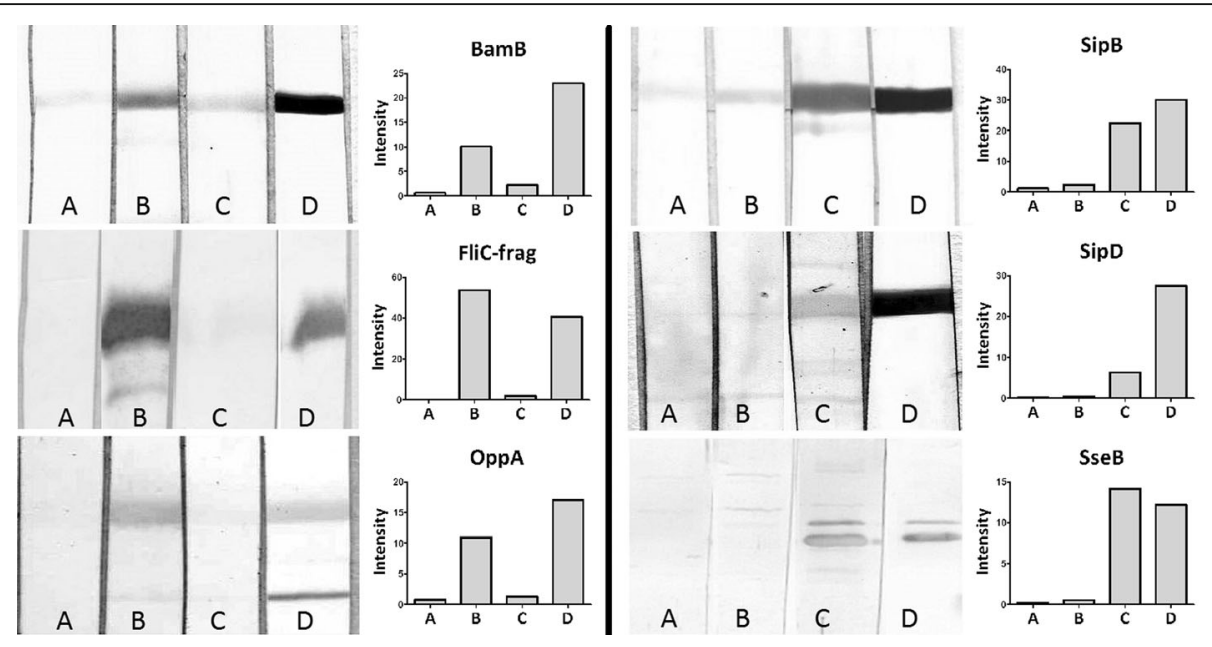

Fig. $\mathbf{2}$ Western blots of recombinant proteins with sera. Western blots with respective density graphs of purified recombinant proteins with $\mathbf{A}-$ control sera, B - post-vaccination sera, C - post-infection sera, and $\mathbf{D}$ - with anti-HisTag Ab

can see the increase of anti-SipB IgG level from the third week of infection but not significant differences for antiFliC antibodies throughout the infection experiment (Fig. 4a, b). In contrary, the vaccination does not induce anti-SipB antibody production but anti-FliC IgGs are detectable after 14 days that is the time of revaccination (Fig. 4d, e). For comparison, we measured the level of postinfection and post-vaccination antibodies against $\mathrm{O}$ antigens - the outer segments of surface lipopolysaccharides using commercially available ELISA kit (Fig. 4c, f). As expected, we found a similar pattern of immunoglobulin G production in both, infected and vaccinated groups. Additionally, a concentration of IgM antibodies against SipB protein did not exceed positive threshold after the infection. Anti-LPS IgMs were detectable on day 7 and 14 in two and four pigs respectively (see Additional file 1: Figure S2).

\section{Discussion}

Although the vaccination of animals against bacterial pathogens helps to moderate the severity of infection and is widely used in veterinary medicine, there are several issues that have to be solved during the development process and before introducing such vaccines to the market. Besides the induction of humoral and cellular immunity - the protectivity itself, in many cases, we must ensure a proper distinction between animals after the natural infection and vaccinated animals - i.e. DIVA vaccines [12]. We believe that using a vaccine with differentiability of infected and vaccinated animals enable establishment of Salmonella control programs in pig herds. This goal is currently not possible using the only licensed live attenuated vaccine [7]. In a case of Salmonella DIVA vaccines, several groups followed the path of a negative marker vaccine $[7,8]$, which uses a vaccine strain with a deleted gene for an antigenic protein. Host antibodies against this protein are thus found only in naturally infected animals and are absent in vaccinated animals. However, using live attenuated genetically modified Salmonella vaccine strains in pig herds is not straightforward. We proved inactivated Salmonella vaccine efficiently protects suckling piglets against infection [9] and further developed a DIVA method whereby there is no need for using a live genetically modified organism as an antigen for a vaccine. To develop DIVA assay, we decided to use a novel approach
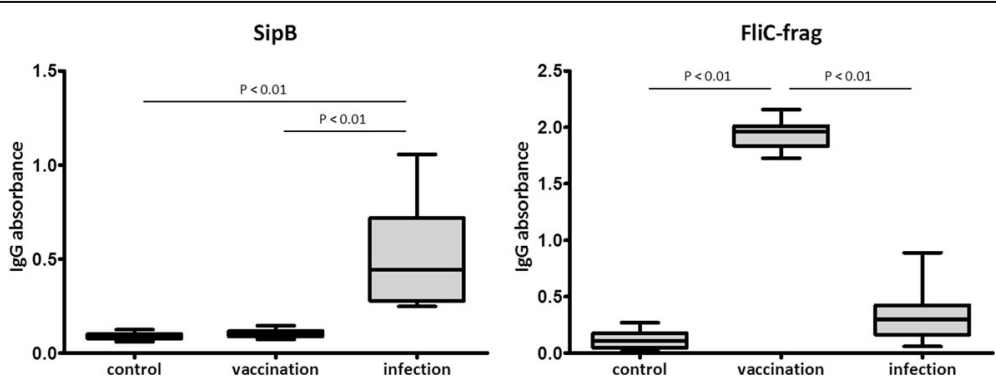

Fig. 3 ELISA assay of recombinant proteins. Graphs of IgG absorbance from ELISA assays of two recombinant proteins incubated with individual sera of control animals $(N=12)$, STM infected animals $(N=12)$ and animals after vaccination with STM-based vaccine $(N=12)$ 

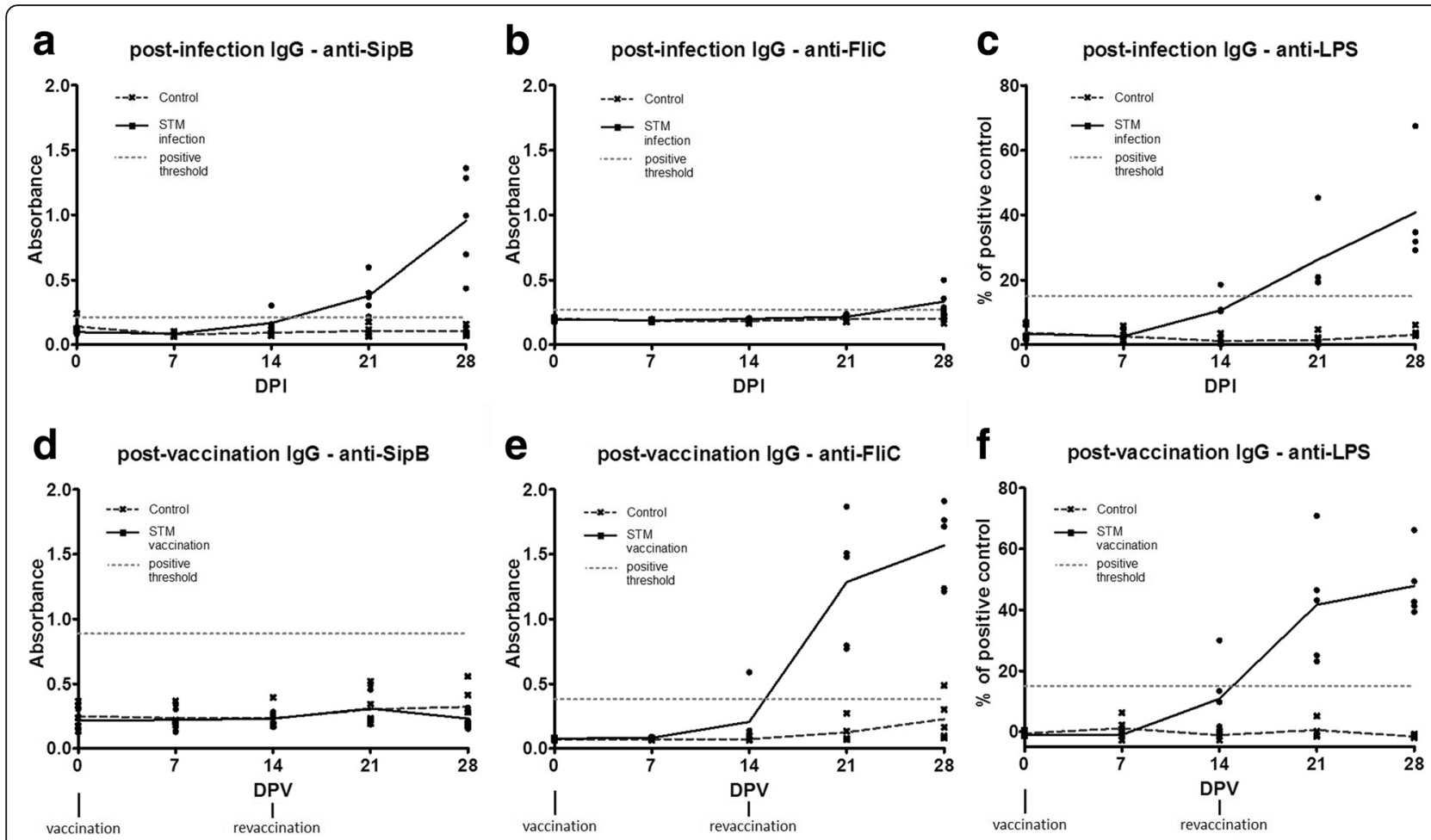

Fig. 4 Time course of antibody response. The time course of IgG antibody response to SipB and FliC-frag proteins after the infection (a, b) or vaccination (d, e). For comparison, graphs $\mathbf{c}$ and $\mathbf{f}$ show antibody response to O-antigens of Salmonella LPS. The connecting lines determine the average of five animals randomly selected from each group

based on examination of the natural antibody response against Salmonella Typhimurium after the infection and after the vaccination with a Salmonella Typhimuriumbased inactivated vaccine. We took advantage of that Salmonella Typhimurium undergoes several different stages during the infection according to its localization and to the host immune response. Following these conditions, bacteria change the expression of virulence and protecting proteins $[13,14]$ that are subsequently exposed to the immune system.

Besides proven protectivity of the used vaccine in suckling piglets [9], we found that antibodies produced after the infection, are indeed different from those after the vaccination. From the immunoaffinity chromatography and mass spectrometry, we selected the following candidate proteins - SipB, SipC, MetQ, OppA, Bam and SurA. Then, we added some proteins that were not determined by the aforementioned method, but are known to be involved in the infection process as they belong to the SPI-1 or SPI-2 locus - SipD, SseB and PrgI and a highly immunogenic flagellar protein FliC.

Proteins SipB, SipC and SipD are representatives of the SPI-1 system which is responsible for invading the host cells. It is well established that these proteins form a tip of a needle structure of the type III secretion system complex that interacts with the eukaryotic cells $[13,15]$. Protein
SseB encoded by the SPI- 2 locus acts as a translocator that mediates translocation of effector proteins into the host cells and promotes bacterial survival [16]. These proteins are therefore crucial for virulence and are candidates for inducing the antibody response that was also confirmed in this work. On the other hand, when Salmonella Typhimurium was cultivated in vitro in BHI culture medium as a vaccine batch, these proteins were not expressed in amounts able to induce antibody production. SPI-1 proteins were nevertheless found in high amounts when Salmonella had grown in LB medium. This medium is therefore not applicable for a vaccine batch preparation because it could induce production of the same antibodies as after a natural infection. Nutrient-limiting conditions provided by LB broth partially resemble in vivo environment and trigger SPI-1 proteins expression, while rich BHI medium does not induce production of Salmonella virulence factors. Jaradat and Bhunia [17] suggested a similar explanation as gained from their results in different growth conditions of Listeria monocytogenes, stating that bacteria produce more virulence factors in a nutritionally poor milieu in order to adapt to the new environment. On the other hand, bacterial virulence factors seem to be good candidates for antibody response induction and therefore for the vaccine protectivity in general. However, we showed that the efficacy of our vaccine prepared 
from a culture grown in a medium not inducing the expression of SPI proteins is high [9].

In spite of the fact that flagellin has powerful immunostimulatory properties and may play a role as a potent adjuvant in a variety of anti-bacterial vaccines [18-20], it was also shown that expression of flagellar proteins is selectively repressed when Salmonella is located intracellularly during the infection [21, 22]. The flagellum as an extracellular structure on the surface of a bacterium could be a good candidate as a post-vaccination antigen if an appropriate growth medium capable of promoting the expression of flagellar proteins is used. This was proved for FliC protein fragment by western-blot and ELISA but interestingly not by PSM spectra of immunoaffinity eluates. We speculate this was caused by overall high amount of anti-FliC antibodies in all samples and the capacity of immunoaffinity columns was probably saturated. Only western-blot and ELISA were able to show much higher anti-FliC antibody level in postvaccination sera than in post-infection and control sera. PSM for FliC in control sample was probably caused by cross-reactivity of anti-flagellar antibodies induced by other bacteria in control animals. The other possible explanation is very high expression of FliC protein in cultures and possible unspecific binding in the chromatography column. Proteins BamB (Outer membrane protein assembly factor) and OppA (Periplasmic oligopeptide-binding protein) are located on the outer membrane and the periplasm, respectively, which makes them easy targets for the immune system. Although both proteins are involved in transmembrane transport (OppA as a permease [23] and BamB as a part of the outer membrane protein assembly complex involved in insertion of beta-barrel proteins [24]), their role in the infection is not known. Interestingly, both proteins are much less immunogenic during infection than after vaccination. This finding makes them together with FliC good candidates for serological differentiation between infected and vaccinated animals.

After successful isolation of recombinant proteins, we tested them using western blot and ELISA with postinfection, post-vaccination and control serum samples to prove their ability to distinguish between infected and vaccinated animals. Proteins SseB, SipB and SipD were confirmed to be highly immunogenic during the infection. This is in agreement with previous findings [25, 26]. The level of antibodies against these proteins, measured as a density of bands on western blots, was, at least, ten times higher in a group of infected animals than in a group of control or vaccinated animals. On the other hand, proteins BamB, OppA and FliC-frag were confirmed to be highly immunogenic during the vaccination of pigs. The level of antibodies against these proteins was, at least, ten times higher in a group of vaccinated animals than in a group of control or infected animals. As in the case of FliC, there is a partial discrepancy with immunoaffinity chromatography results in the case of SseB protein when it showed a similar level of binding to post-infection and post-vaccination antibodies. However, it can be explained by low expression of SseB in cultivation media and thus possible incomplete saturation of all specific antibodies present in the chromatography column.

Finally, proteins SipB and FliC-frag were selected for ELISA assays to examine the interaction with 36 porcine serum samples. All 12 post-infection sera exhibited higher absorbance with the SipB protein than 12 control and 12 post-vaccination sera. In contrast, all 12 postvaccination sera exhibited higher absorbance with the FliC-frag protein than 12 control and 12 post-infection sera. All differences were statistically significant ( $P$ $<0.01)$. The time-dependent onset of IgG antibody levels against SipB after the infection, and against FliC after the vaccination imitates the increase of IgG against surface lipopolysaccharide. These two proteins are thus considered as useful antigens for serological differentiation of infected and vaccinated animals.

In general, induction of different protein expression profiles, their potential exposure to the immune recognition machinery and subsequent specific antibody production can be conveniently applied for the differentiation between infected and vaccinated animals without any intervention into the genome of an organism used as a vaccine strain.

\section{Conclusions}

Using antibodies from previously vaccinated and infected animals and using immunoaffinity chromatography and mass spectrometry tools, we identified Salmonella proteins that induce post-infection and postvaccination specific antibody production. Gene cloning and recombinant protein expression techniques were used for antigen preparation that allows serological distinction between infected animals and animals vaccinated with a Salmonella-based inactivated vaccine.

This approach can be utilized in many other challenges where DIVA vaccine and a subsequent serological assay are required, especially when genetic modification of a vaccine strain is not desirable.

\section{Additional files}

Additional file 1: Figure S1. Recombinant proteins expressions. Coomassie-stained gels and western blots of recombinant proteins. Time scale expression is shown in hours post induction (0-5 h, ON - overnight). Arrows indicate the expected molecular weight of a product. Figure S2. $\lg M$ antibody response. The time course of IgM antibody response to SipB protein and to Salmonella LPS after the infection. The connecting 
lines determine the average of five animals randomly selected from each group. (PDF $434 \mathrm{~kb}$ )

Additional file 2: Identified Salmonella proteins from different media. Mass spectrometry identification of Salmonella proteins and a comparison of expression between two cultivation media (LB vs BHI). (XLSX $433 \mathrm{~kb})$

Additional file 3: Identified proteins from immunoaffinity chromatography. Mass spectrometry identification of Salmonella proteins bound to control, post-infection or post-vaccination serum. Immunoaffinity chromatography was made in triplicates. (XLSX $913 \mathrm{~kb}$ )

\section{Abbreviations}

BHI: Brain-heart infusion; CFU: Colony-forming unit; DIVA: Differentiation of infected and vaccinated animals; FASP: Filter-aided sample preparation; FPLC: Fast protein liquid chromatography; HRP: Horseradish peroxidase; LB: Lysogeny broth; LPS: Lipopolysaccharide; MS: Mass spectrometry; PSM: Peptide spectrum match; PVDF: Polyvinylidene difluoride; SPI: Salmonella pathogenicity island; TMB: 3,3',5,5'-tetramethylbenzidine: UHPLC: Ultra-high pressure liquid chromatography

\section{Acknowledgments}

The authors wish to thank Dr. Ludmila Faldikova for correcting and proofreading of the manuscript.

\section{Funding}

This study was supported by the Ministry of Agriculture of the Czech Republic (QJ1210115, RO0516) and the Ministry of Education, Youth and Sports of the Czech Republic (LO1218 from the MEYS of the CR under the NPU I program).

\section{Availability of data and material}

The datasets of proteomic results from culture media comparison and immunoaffinity chromatography are included within Additional files 2 and 3 of the article. All other data generated or analysed during this study are included in this published article. Proteins mentioned in patent 305077 can be used in serological methods for experimental purposes.

\section{Authors' contributions}

JG carried out immunoaffinity chromatography, mass spectrometry, and the cloning and wrote the first and the final draft of the manuscript. HK performed all ELISA assays including their validation. MK contributed to culture media testing and in vaccination design. KK designed the serological assays. RT did the isolation and the purification of recombinant proteins. AO participated in the experimental infection and vaccination and sample collection. MF helped to design the animal experiments, obtained financial support and took part in the revising of the manuscript. JM was responsible for the whole project, generated the working hypothesis, designed the experiments, obtained financial support, revised the draft and wrote the final version of the manuscript. All authors read and approved the final manuscript.

\section{Competing interests}

Using SseB, Gene ID: 1252916; SipB, Gene ID: 1254408; SipD, Gene ID: 1254406; FliC, Gene ID: 1253480 and BamB, Gene ID: 1254042 proteins in serological methods differentiating infected and vaccinated pigs is covered by Czech patent number 305077. Except of Martin Faldyna, all authors are holders of 305077 patent rights. Marcel Kosina is an employee of Bioveta company which potentially may have a commercial benefit from results of this work. The authors declare that they have no competing interests.

\section{Consent for publication}

Not applicable

\section{Ethics approval}

All animal experiments were performed at animal barrier facility at Veterinary Research Institute. The experimental protocol was approved by the Branch Commission for Animal Welfare of the Ministry of Agriculture of the Czech Republic (Ref. No. MZE 921). Based on current legislation, the Branch Commission for Animal Welfare of the Ministry of Agriculture of the Czech
Republic has exclusive rights to approve infection experiments on animals in the Czech Republic (Act No 246/1992 Coll.).

\section{Author details}

'Department of Immunology, Veterinary Research Institute, Hudcova296/70, 62100 Brno, Czech Republic. '2Department of Experimental Biology, Faculty of Science, Masaryk University, Kotlarska267/2, 61137 Brno, Czech Republic. ${ }^{3}$ Bioveta a. S., Komenskeho212/12, 68323 Ivanovice na Hane, Czech Republic. ${ }^{4}$ Department of Virology, Veterinary Research Institute, Hudcova296/70, 62100 Brno, Czech Republic. ${ }^{5}$ Faculty of Veterinary Medicine, University of Veterinary and Pharmaceutical Sciences, Palackeho 1/3, 61242 Brno, Czech Republic.

Received: 21 April 2016 Accepted: 3 November 2016

Published online: 11 November 2016

\section{References}

1. Anonymous. Analysis of the baseline survey on the prevalence of Salmonella in holdings with breeding pigs in the EU, 2008 - Part A: Salmonella prevalence estimates. EFSA J. 2009;7:1377.

2. van Oirschot JT. Diva vaccines that reduce virus transmission. J Biotechnol. 1999:73(I2-3):195-205.

3. Vico JP, Engel B, Buist WG, Mainar-Jaime RC. Evaluation of three commercial enzyme-linked immunosorbent assays for the detection of antibodies against Salmonella spp. in meat juice from finishing pigs in Spain. Zoonoses Public Health. 2010;57 Suppl 1:107-14.

4. Leyman B, Boyen F, Van Parys A, Verbrugghe E, Haesebrouck F, Pasmans F. Salmonella Typhimurium LPS mutations for use in vaccines allowing differentiation of infected and vaccinated pigs. Vaccine. 2011;29(20):3679-85.

5. Zariri A, van der Ley P. Biosynthetically engineered lipopolysaccharide as vaccine adjuvant. Expert Rev Vaccines. 2015;14(6):861-76.

6. Arenas J. The Role of Bacterial Lipopolysaccharides as Immune Modulator in Vaccine and Drug Development. Endocr Metab Immune Disord Drug Targets. 2012;12(3):221-35.

7. Selke M, Meens J, Springer S, Frank R, Gerlach GF. Immunization of pigs to prevent disease in humans: construction and protective efficacy of a Salmonella enterica serovar Typhimurium live negative-marker vaccine. Infect Immun. 2007;75(5):2476-83.

8. Bearson BL, Bearson SMD, Kich JD. A DIVA vaccine for cross-protection against Salmonella. Vaccine. 2016;34(10):1241-6.

9. Matiasovic J, Kudlackova H, Babickova K, Stepanova H, Volf J, Rychlik I, Babak $\checkmark$, Faldyna M. Impact of maternally-derived antibodies against Salmonella enterica serovar Typhimurium on the bacterial load in suckling piglets. Vet J. 2013;196(1):114-5.

10. Wiśniewski JR, Zougman A, Nagaraj N, Mann M. Universal sample preparation method for proteome analysis. Nat Methods. 2009;6(5):359-62.

11. Jacobson $\mathrm{RH}$. Factors in selecting serum samples for use in determining the positive/negative threshold (cut-off) in ELISA. Diagnosis and Epidemiology of Animal Disease in Latin America 1998. IAEA-TECDOC-1055, pp. 25-28. http://www-pub.iaea.org/books/IAEABooks/5352/Diagnosis-andEpidemiology-of-Animal-Diseases-in-Latin-America.

12. Pasick J. Application of DIVA vaccines and their companion diagnostic tests to foreign animal disease eradication. Anim Health Res Rev. 2004;5(2):257-62.

13. Mirold S, Ehrbar K, Weissmüller A, Prager R, Tschäpe H, Rüssmann H, Hardt WD. Salmonella host cell invasion emerged by acquisition of a mosaic of separate genetic elements, including Salmonella pathogenicity island 1 (SPI1), SPI5, and sopE2. J Bacteriol. 2001;183(7):2348-58.

14. McGhie EJ, Brawn LC, Hume PJ, Humphreys D, Koronakis V. Salmonella takes control: effector-driven manipulation of the host. Curr Opin Microbiol. 2009;12(1):117-24

15. Marlovits TC, Stebbins CE. Type III secretion systems shape up as they ship out. Curr Opin Microbiol. 2010;13:47-52.

16. Waterman SR, Holden DW. Functions and effectors of the Salmonella pathogenicity island 2 type III secretion system. Cell Microbiol. 2003;5(8):501-11.

17. Jaradat ZW, Bhunia AK. Glucose and nutrient concentrations affect the expression of a 104-kilodalton Listeria adhesion protein in Listeria monocytogenes. Appl Environ Microbiol. 2002;68(10):4876-83.

18. Smith KD, Andersen-Nissen E, Hayashi F, Strobe K, Bergman MA, Rassoulian Barrett SL, Cookson BT, Aderem A. Toll-like receptor 5 recognizes a conserved site on flagellin required for protofilament formation and bacterial motility. Nat Immunol. 2003;4:1247-53. 
19. das Gracas Luna M, Sardella FF, Ferreira LC. Salmonella flagellin fused with a linear epitope of colonization factor antigen I (CFA/I) can prime antibody responses against homologous and heterologous fimbriae of enterotoxigenic Escherichia coli. Res Micribiol. 2000;151:575-82

20. Honko AN, Sriranganathan N, Lees CJ, Mizel SB. Flagellin is an effective adjuvant for immunization against lethal respiratory challenge with Yersinia pestits. Infect Immun. 2006;74:1113-20.

21. Cummings LA, Rassoulian Barrett SL, Wilkerson WD, Fellnerova I, Cookson BT. Flic-specific CD4 ${ }^{+} T$ cell responses are restricted by bacterial regulation of antigen expression. J Immunol. 2005;174(12):7929-38.

22. Lai MA, Quarles EK, López-Yglesias AH, Zhao X, Hajjar AM, Smith KD. Innate immune detection of flagellin positively and negatively regulates Salmonella infection. PLoS One. 2013;8(8):e72047.

23. Hiles ID, Gallagher MP, Jamieson DJ, Higgins CF. Molecular characterization of the oligopeptide permease of Salmonella typhimurium. J Mol Biol. 1987; 195(11):125-42.

24. Hagan CL, Silhavy TJ, Kahne D. $\beta$-Barrel Membrane Protein Assembly by the Bam Complex. Annu Rev Biochem. 2011;80:189-210.

25. Lee S-J, Liang L, Juarez S, Nanton MR, Gondwe EN, Msefula CL, Kayala MA, Necchi F, Heath JN, Hart P, Tsolis RM, Heyderman RS, Mac Lennan CA, Felgner PL, Davies DH, McSorley SJ. Identification of a common immune signature in murine and human systemic Salmonellosis. Proc Natl Acad Sci. 2012;109(13):4998-5003.

26. Desin TS, Mickael CS, Lam PK, Potter AA, Köster W. Protection of epithelial cells from Salmonella enterica serovar Enteritidis invasion by antibodies against the SPI-1 type III secretion system. Can J Microbiol. 2010;56(6):522-6.

\section{Submit your next manuscript to BioMed Central} and we will help you at every step:

- We accept pre-submission inquiries

- Our selector tool helps you to find the most relevant journal

- We provide round the clock customer support

- Convenient online submission

- Thorough peer review

- Inclusion in PubMed and all major indexing services

- Maximum visibility for your research

Submit your manuscript at www.biomedcentral.com/submit

C) Biomed Central 